\title{
Ambient Assisted Living
}

\section{Wie neue Technologien pflegende Angehörige entlasten können}

\author{
Tibor Vetter
}

Tibor Vetter studierte Medieninformatik an der Akademie für Datenverarbeitung in Böblingen und Soziale Arbeit an der Hochschule Esslingen. Seit Oktober 2009 arbeitet er als Projektmanager beim Wohlfahrtswerk für BadenWürttemberg in Stuttgart und ist dort für das Vorhaben »easyCare« tätig. E-Mail tibor.vetter@wohlfahrtswerk.de
Häusliche Pflege wird nach wie vor zum größten Teil von Angehörigen - und vor allem von Frauen - geleistet. Zwar gibt es ein breites Spektrum an Beratungs- und Unterstützungsangeboten, doch können diese von den betroffenen Angehörigen kaum in Anspruch genommen werden, weil sie nicht bekannt sind, zusätzlichen Organisationsaufwand erfordern oder für die individuelle Lebenssituation nicht passen. Mit Konzepten des Ambient Assisted Living versucht man deshalb, neue technisch unterstützte Assistenzsysteme und bedarfsgerechte Dienstleistungen zu erproben.

In Deutschland vollzieht sich ein dramatischer demografischer Wandel. Die Menschen werden aufgrund einer stetigen Verbesserung der Gesundheitsversorgung immer älter. Ein seit Jahren zu verzeichnender Geburtenrückgang beschleunigt die Entwicklung der "Vergreisung " in Deutschland zusätzlich. Laut Bundespflegestatistik 2007 sind bereits heute rund 2,25 Millionen Menschen im Sinne des Pflegeversicherungsgesetzes pflegebedürftig. Der überwiegende Teil (ca. 70\%) der in häuslicher Pflege versorgten Menschen wird ausschließlich von Angehörigen betreut (vgl. Abb. 1). Damit ist die Familie nach wie vor der größte Pflegedienst Deutschlands.

Dabei steht außer Frage, dass nicht nur die meisten zu Pflegenden häusliche Betreuung mit bekannten Bezugspersonen vorziehen, sondern dies auch volkswirtschaftlich die bevorzugte Lösung darstellt, da ambulante Pflege zu Hause als kostengünstiger gegenüber dem Pflegeheim gilt. Das oft zitierte "ambulant vor stationär « drückt dabei » einerseits die Intention des Gesetzgebers aus, die familiale Pflegebereitschaft zu fördern und anderseits die wohlfahrtsstaatliche Vorstellung, dass man faktisch immer noch auf die Stabilität eines Familiennetzwerkes informeller Helfer zurückgreifen kann« (Meyer 2006).
Für Familienangehörige stellt die Pflege jedoch eine erhebliche Belastung dar. Sie müssen die erkrankungsbedingten psychischen Veränderungen und physischen Beeinträchtigungen eines geliebten Menschen emotional verarbeiten, körperlich schwere Pflegearbeit erbringen und sich mit finanziellen und rechtlichen Belangen auseinandersetzen. Oftmals ist daher nicht der Gesundheitszustand der Pflegebedürftigen, sondern die Überforderung der Angehörigen Auslöser für die Suche nach Alternativen.

\section{Hilfe durch Dienstleistungen und Technik}

Ein Ansatz in jüngster Zeit sind technologische Entwicklungen des Ambient Assisted Living (AAL). Man versteht darunter technisch unterstützte Assistenzsysteme und bedarfsgerechte Dienstleistungen, die bei Menschen mit körperlichen oder kognitiven Einschränkungen zur Erhaltung ihrer Selbständigkeit im eigenen Heim beitragen. (1) In Deutschland werden seit 2009 unter dem Titel »Altersgerechte Assistenzsysteme für ein gesundes und unabhängiges Leben « vom Bundesministerium für Bildung und Forschung 18 Projekte gefördert. Neue Technologien und soziales Umfeld miteinander zu verbinden ist Ziel dieses interdisziplinären Forschungsprogramms. Vier wichtige Anwendungsfelder für Ambient-AssistedLiving-Technologien kristallisieren sich heraus (BMBF 2008):

- Gesundheit und Home Care: Einsatz intelligenter Assistenzsysteme zur Unterstützung von Prävention und Rehabilitation im häuslichen Bereich, beispielsweise durch Teleüberwachung, Pflegeunterstützung, elektronische Hilfen und Roboter.

- Sicherheit und Privatsphäre: Einsatz von Sicherheitssystemen zur frühzeitigen Gefahrenerkennung (vergessenes Ausschalten von Geräten wie Herd 


\section{„ECKDATEN“ PFLEGESTATISTIK 2007}

\section{2,25 Millionen Pflegebedürftige insgesamt}

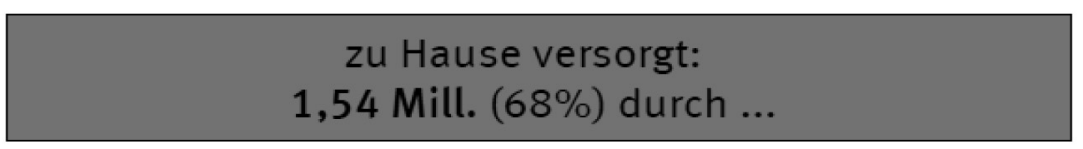

in Heimen versorgt: $709.000(32 \%)$
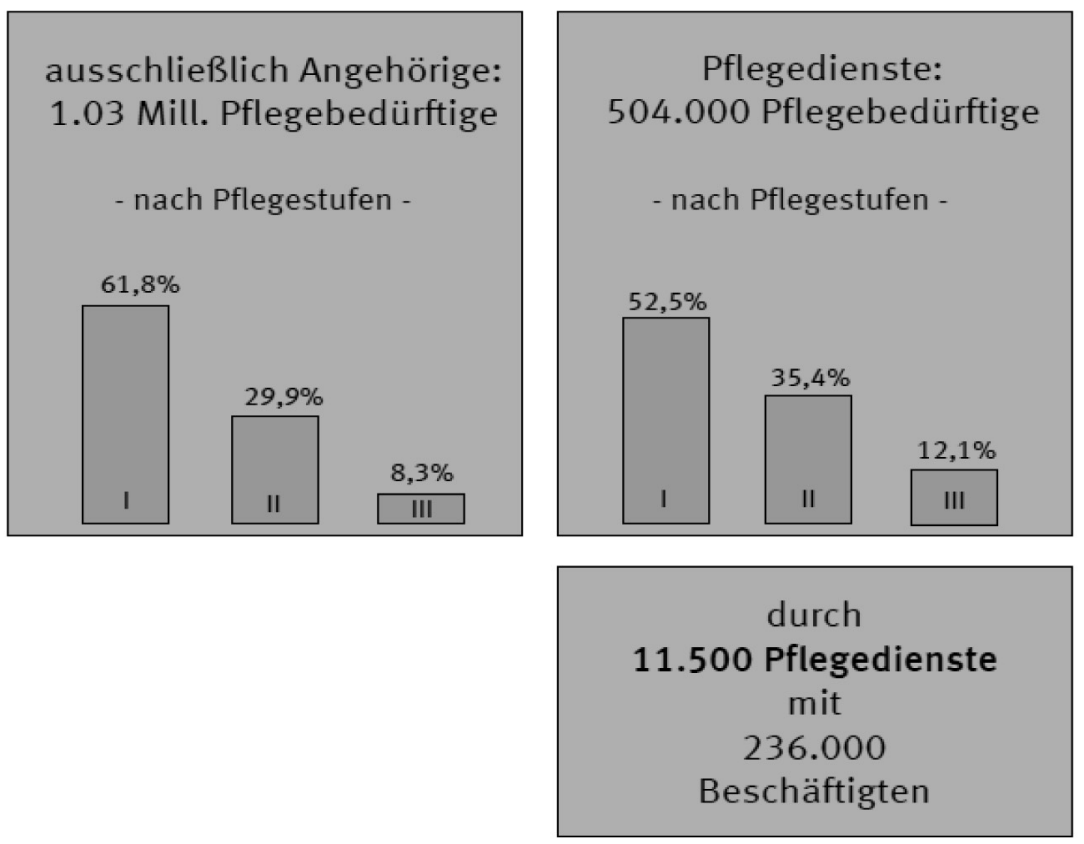
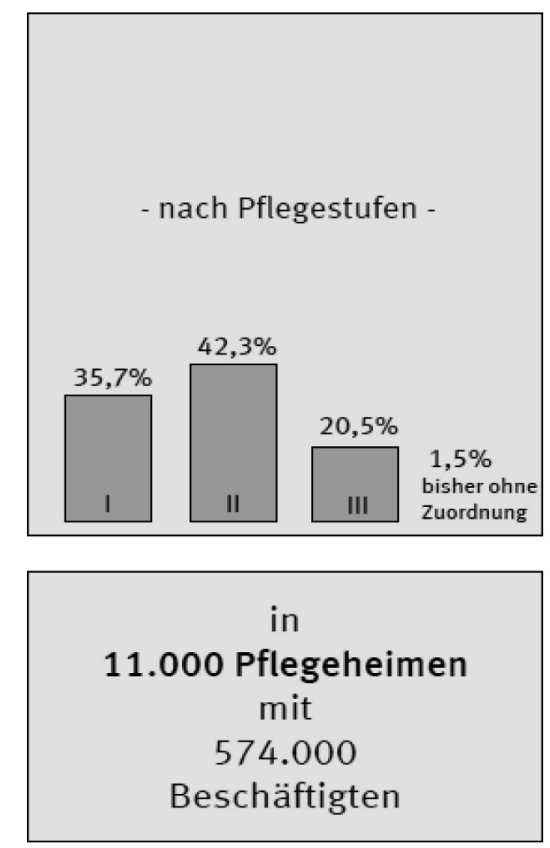

Abb. 1: Rund 70 Prozent der in häuslicher Pflege versorgten Menschen wird ausschließlich von Angehörigen betreut. Quelle: Statistisches Bundesamt 2008.

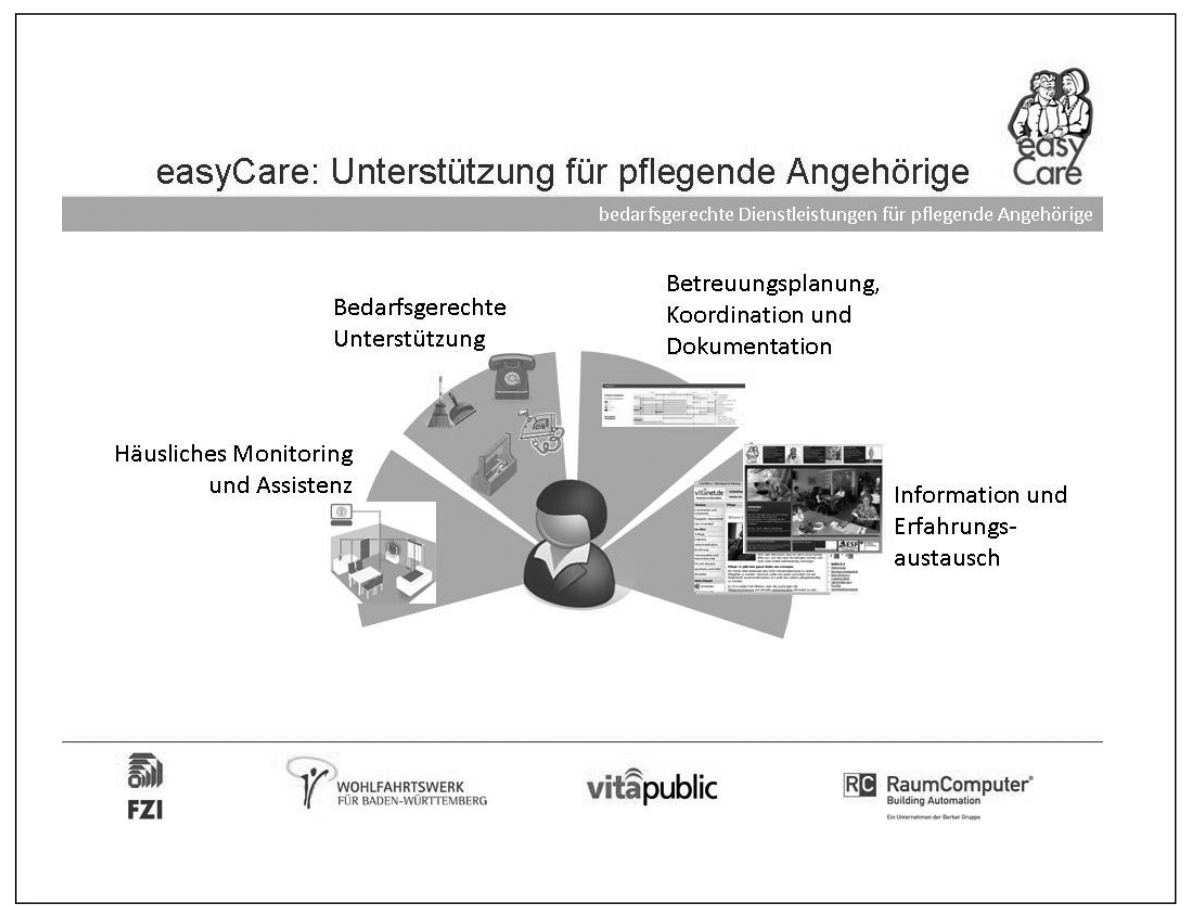

Abb. 2: Die Unterstützung für pflegende Angehörige basiert im Projekt »easyCare« vor allem auf vier Säulen. oder Bügeleisen) oder von Erinnerungsfunktionen (z.B. vergessene Medikamenteneinnahme oder Termine). Neben diesen rein technisch basierten Lösungsansätzen sollen auch einfache Umbauten (rutschfeste Teppiche, Haltegriffe in Badewannen) dazu beitragen, das Sicherheitsbedürfnis zu befriedigen und die Mobilität älterer Menschen zu erhöhen.

- Versorgung und Haushalt: Verknüpfung einzelner, bereits im Haushalt vorhandener technischer Gerätschaften (z.B. Heizung, Klimaanlage, Licht oder Alarmanlage) zu einem »intelligenten, mitdenkenden « Gesamtsystem.

- Soziales Umfeld: Einsatz von innovativen Informations- und Kommunikationstechnologien zur Unterstützung und Erleichterung der sozialen Teilhabe. Lösungen aus dem Bereich der Videokommunikation oder des Internets ermöglichen älteren, in ihrer Motorik eingeschränkten Menschen auch bei 


\section{Was die Technik können muss}

Unter Ambient Assisted Living versteht man technisch unterstützte Assistenzsysteme und bedarfsgerechte Dienstleistungen, die bei Menschen mit körperlichen oder kognitiven Einschränkungen zur Erhaltung ihrer Selbständigkeit im eigenen Heim beitragen. Ambient-Assisted-Living-Technologien müssen:

- zuverlässig, leicht installierbar und einfach zu bedienen sein

- flexibel und nachrüstfähig sein, so dass sie auch in bestehende Wohnungen eingebaut werden können

- Sicherheit gewährleisten und die persönliche Autonomie der Nutzer(innen) schützen

- adaptierbar an konkrete Alltagszusammenhänge und Arbeitsprozesse sein und sich an Veränderungen der Lebensumstände und Arbeitsprozesse anpassen können

- Kommunikation zwischen allen Beteiligten erleichtern

- ästhetischen Ansprüchen der Zielgruppen genügen und nicht stigmatisieren

- kostengünstig sein oder von Kranken- und Pflegekassen unterstützt werden

Quelle: BMBF/VDE 2010

räumlicher Distanz neue Kontakte zu knüpfen oder aufrecht zu erhalten (Verwandte, Freunde).

Noch scheitert manche Anwendung technologiegestützter Assistenzsysteme an Akzeptanzhürden der Endanwender, teils wegen Schwierigkeiten bei der altersgerechten Bedienung, wegen einer mit den Diensten oder Produkten assoziierten Stigmatisierung, aus Kostengründen oder Unwissenheit. Selbst Experten, die Beratung und Case Management leisten oder Multiplikatoren sind, empfinden ein großes Know-how-Defizit.

\section{Pflegende Angehörige sind weiblich und berufstätig}

$\mathrm{Ob}$ und wie lange pflegebedürftige Menschen in ihrer bevorzugten häuslichen Umgebung trotz gesundheitlicher Beeinträchtigungen verbleiben können, hängt neben ihrer Pflegebedürftigkeit in hohem Maße von der Situation pflegender Angehöriger ab.

»Betreuung und Pflege alter Menschen im Privathaushalt ist Frauensache. (Jentzsch 2004) Es sind vor allem Ehefrauen, Töchter und Schwiegertöchter, oft selbst in fortgeschrittenem Alter, mehrfach belastet durch eigene Familie oder Berufstätigkeit, die das Pflegereservoir bilden, so das Ergebnis mehrerer Studien zur Situation pflegender Angehöriger (vgl. Tabelle »Pflegende Angehörige $\ll)$.

Europaweit übernehmen 76 Prozent die Hauptlast der Pflegeaufgaben zu Hause (Döhner et al. 2007). Das Durch- schnittsalter der Pflegepersonen steigt in Deutschland kontinuierlich an. Pflegende Angehörige sind heute im Mittel 60 Jahre (1997: 57 Jahre) alt. Eine Mehrzahl von 54 Prozent ist zwischen 40 und 64 Jahren.

Ein weiterer Trend ist die Zunahme der Erwerbstätigkeit. »Über die Hälfte der Angehörigen sind berufstätig und arbeiten im Durchschnitt 31,8 Wochenstunden ", so das Ergebnis einer 2009 vorgestellten Studie (Runde et al. 2009). Das Ausmaß der Erwerbsbeteiligung hängt dabei in hohem Maße vom Grad der Pflegebedürftigkeit sowie vom Belastungserleben ab. Belastend wirken neben Alter und Erwerbsbeteiligung auch Pflegedauer, Umfang der Pflegeaufgaben und weitere, sehr unterschiedliche Problembereiche:

- Fehlende Sicherheit bezüglich der Situation und des Zustands der Pflegebedürftigen: In getrennten Haushalten lebende Angehörige haben nur einen eingeschränkten Einblick in die Situation der Pflegebedürftigen. Notfälle (z.B. Stürze) oder Zustandsveränderungen (z.B. nächtliche Schlafstörungen oder fortschreitende Demenz) mit damit verbundenen Gefahren werden nicht oder erst spät erkannt. Die damit verbundene Unsicherheit stellt für die Pflegenden eine große psychische Belastung dar.

- Fehlende Anleitung und Organisationshilfen: In schwierigen Situationen stehen pflegende Angehörige oft alleine da. Sie sind für Pflegetätigkeiten selten ausreichend ausgebildet. Bei Problemen fehlt es ihnen an situativer Anleitung und professionellem Coaching. Anders als bei professionellen Pflegenden fehlen Nachschlagewerke, Ablagestrukturen und personenübergreifende Terminplanungswerkzeuge zur Koordination der Betreuung und Pflege.

- Mangelnder Austausch: Oft sind Rat und emotionale Unterstützung von Menschen in einer ähnlichen Lage eine wichtige Hilfe für pflegende Angehörige. Wohl nur diese Menschen haben ein vergleichbares Verständnis der Situation, nicht nur auf der fachlichen, sondern auch auf der emotionalen Ebene.

- Fehlende bedarfsgerechte Unterstützung: Angehörige sehen heute meist nur die Wahl zwischen professionellen, unter Zeitdruck geleisteten Diensten oder der selbst geleisteten Pflege. Angebote zur passgenauen Unterstützung existieren meist nicht.

Zwar gibt es heute ein breites Spektrum an Beratungs-, Betreuungs- und Versorgungsangeboten, doch werden diese von den Betroffenen kaum in Anspruch genommen. Nur 16 Prozent der Hauptpflegepersonen greifen regelmäßig auf Beratungs- oder Unterstützungsangebote zurück. Im Gegensatz dazu nimmt die Hälfte der pflegenden Angehörigen die Hilfen und Angebote der im Gesundheitssystem vorhandenen Beratungs- und Betreuungsstrukturen nicht an (Schneekloth/Wahl 2005).

Dies mag damit zusammenhängen, dass die benötigte Unterstützung analog zu heterogenen Pflegesituationen von Familie zu Familie variiert. Viele Angebote wirken häufig nur punktuell und pflegende Angehörige müssen von vielen verschiedenen Seiten Unterstützung einholen. Fehlende Kenntnis, wo es welche Hilfe gibt, die man sich zu alledem noch selbst organisieren muss, kann in prekären Situationen schnell das Gefühl einer Überforderung auslösen - erst recht, wenn es an Anleitung, Organisationshilfen und Austausch fehlt.

\section{Den Helfern helfen: das Projekt easyCare}

An diesen Konstellationen pflegender Angehöriger knüpft das Projekt »easyCare« an. (2) Ein zentrales Internetportal liefert pflegenden Angehörigen relevante fachliche Informationen, die gezielte Anleitungen beinhalten und multimedial aufbereitetet die Pflege- und Betreuungs- 
tätigkeit erleichtern soll. Virtuelle Diskussionen in Chatrooms oder Foren des Portals fördern den Informations- und Erfahrungsaustausch zwischen pflegenden Angehörigen, Anteilnahme und Auseinandersetzung mit psychischen Belastungen aus der Pflegetätigkeit. Für Pflegeberater, die die easyCare-Plattform nutzen wollen, werden Softwarekomponenten zur Verfügung gestellt, die ihnen eine Fallklärung und Hilfeplanung erstellen helfen.

Durch Kontaktaufnahme über die Plattform kann Hilfe unterschiedlicher Art vermittelt werden, was im Projektrahmen regional eng begrenzt erprobt wird (vgl. Abb. 2). Hier geht es auch darum, beispielsweise bei Krankheit oder berufsbedingtem plötzlichem Ausfall der Pflegeperson zeitnah individuelle Lösungen zu finden. Im Rahmen der individuellen Hilfeanfragen von Pflegehaushalten besteht für eine begrenzte Zahl zudem die Möglichkeit, häusliche Assistenzsysteme zu installieren. Damit sollen Angehörige die nötige Sicherheit zur Selbstversorgungskompetenz der Person mit Assistenzbedarf erlangen. Im Projekt sind drei Module abrufbar: Das Sammeln von Informationen (z.B. Nutzung von Haushalts- und anderen Geräten), die Analyse gesammelter Informationen und Alarmierung bei Erkennen einer Notfallsituation.

Die Mehrdimensionalität des Projekts soll besonders häufige Problemkonstellationen erkennbar machen, und zeigen, wo technologische Unterstützung besondere Wirksamkeit erreichen kann. Letztlich dient das Projekt auch der Entwicklung tragfähiger Geschäftsmodelle, bei denen unterschiedliche Akteure und Finanzierungsansätze miteinander kombinierbar sein sollen. Denn von den privaten Haushalten wird man gegebenenfalls steigende Technologie-Affinität, aber nur eine begrenzte Preisakzeptanz für Informations-, Beratungs- und Vermittlungsdienste erwarten dürfen.

\section{Zusammenfassung}

Die demografische Entwicklung hat enorme ökonomische und sozialstrukturelle Auswirkungen, die Wissenschaft, Gesellschaft und Politik schon heute vor große Herausforderungen stellt. Insbesondere beim Thema Pflege werden gravierende Veränderungen erwartet, die ei- nen hohen Bedarf an Unterstützung für die zu pflegenden Menschen und deren Helfer anzeigen.

Durch den Einsatz von Ambient-Assisted-Living-Technologien kann eine steigende Unterstützung der Selbstversorgungskompetenz von Menschen mit Assistenzbedarf in ihrer Wohnumgebung einhergehen. Am Beispiel des Projekts easyCare kann aufgezeigt werden, dass altengerechte Assistenzsysteme und bedarfsgerechte Dienstleistungen ein großes Potenzial haben, pflegende Angehörige bei der Pflege und Betreuung zu entlasten, um pflegebedürftigen Menschen ein möglichst langes und selbstbestimmtes Leben in ihrer gewohnten Umgebung zu ermöglichen. Gleichzeitig wird ein erhebliches Einsparpotential für die Sozialsysteme erwartet und ein erfolgversprechender Markt für innovative Technologien und
Dienstleistungen des Ambient-AssistedLiving geschaffen.

Ob jedoch die anfangs erwähnte wohlfahrtsstaatliche Vorstellung vom traditionellen Modell der häuslichen Pflege vor dem Hintergrund einer Zunahme kinderloser und alleinlebender älterer Menschen, der Erwerbstätigkeit, räumlicher Mobilität und individueller Lebensgestaltung auf Dauer tragfähig ist, bleibt abzuwarten.

\section{Anmerkungen}

(1) Vgl. Internet http://www.aal-deutschland.de.

(2) Das Projekt wird gefördert vom Bundesministerium für Bildung und Forschung. Näheres dazu siehe Internet http://www.projekt-easycare.de.

\section{Literatur}

Bundesministerium für Bildung und Forschung (BMBF) (Hg.): AAL. Altersgerechte Assistenzsysteme für ein gesundes und unabhängiges Leben, Berlin 2008. Internet http://www.aal-deutschland.de/deutschland/aal-faltblatt (Stand: 04.10.2010).

BMBF/VDE Innovationspartnerschaft AAL (Hg.): AAL in der alternden Gesellschaft. Anforderungen, Akzeptanz und Perspektiven, AAL Schriftenreihe, Band 2, Berlin und Offenbach 2010.

Döhner, H. et al./Universitätsklinikum Hamburg-Eppendorf (Hg.): Services for Supporting Family Carers of Older Dependent People in Europe - EUROFAMCARE: Characteristics, Coverage and Usage. The National Survey Report for Germany, Hamburg 2007.

Jentzsch, N: Die Betreuung und Pflege alter Menschen durch Angehörige. Befunde zur Bedeutung der Familie für die Gesellschaft aus »ökonomischer « Perspektive, Eichstätt-Ingolstadt 2004.

Meyer, M./Döhner, H. (Hg.): Pflegende Angehörige in Deutschland. Ein Überblick über den derzeitigen Stand und zukünftige Entwicklungen, Band 10, Hamburg 2006.

Runde P./Eichner K./Scheerer, S.: AOK-Trendbericht Pflege II. Entwicklungen in der häuslichen Pflege seit Einführung der Pflegeversicherung, Hamburg 2009.

Schneekloth, U./Wahl, H.-W. (Hg.): Möglichkeiten und Grenzen selbständiger Lebensführung in privaten Haushalten (MuG III). Integrierter Abschlussbericht im Auftrag des Bundesministeriums für Familie, Senioren, Frauen und Jugend. München 2005. Internet http://www.bmfsfj.de/bmfsfj/generator/RedaktionBMFSFJ/Abteilung3/Pdf-Anlagen/mug3-ingetrierter-gesamtbericht, property=pdf, bereich= bmfsfj,sprache $=$ de,rwb=true.pdf (Stand: 04.10.2010).

Statistisches Bundesamt (Hg.): Bevölkerung Deutschlands bis 2060. 12. koordinierte Bevölkerungsvorausberechnung, Wiesbaden 2009. Internet http://www.destatis.de/ jetspeed/portal/cms/Sites/destatis/Internet/DE/Presse/pk/2009/Bevoelkerung/pressebroschuere_bevoelkerungsentwicklung2009,property=file.pdf (Stand: 04.10.2010). Statistisches Bundesamt (Hg.): Pflegestatistik 2007. Pflege im Rahmen der Pflegeversicherung. Deutschlandstatistik, Wiesbaden 2008. Internet http://www.inqa.de/ Inqa/Redaktion/TIKs/Gesund-Pflegen/PDF/pflegestatistik-2007,property=pdf,bereich=inqa,sprache $=$ de,rwb=true.pdf (Stand: 04.10.2010). 
"Sorge ist das bedrückende Gefühl der Unruhe und Angst und die daraus folgende Mühe für das Wohlergehen."

Gerhard Köbler, Juristisches Wörterbuch. 12. Auflage 2003

"Nur selten weist die Bilanz unserer Sorgen ein Defizit auf. "

Phia Rilke, österreichische Schriftstellerin (1851-1931)

»In der Tat entfremdet sich das Individuum

bei jeder gesellschaftlichen Tätigkeit,

deren Ziel nicht ein anderes konkretes Individuum ist. "

Nicolás Gómez Dávila, kolumbianischer Schriftsteller (1913-1994)

"Wie einfach wäre das Leben, wenn sich die unnötigen Sorgen von den echten unterscheiden ließenl « Karl Heinrich Waggerl, österreichischer Schriftsteller (1897-1973)

"In der Schweiz haben sie Nächstenliebe, fünfhundert Jahre Demokratie und Frieden und was ist dabei herausgekommen? Die Kuckucksuhr."

Orson Welles, amerikanischer Filmregisseur (1915-1985)

"Ich glaube, dass in dem Maße, in dem unsere Welt verrückt und unmenschlich zu werden scheint, eine immer größere Zahl von Menschen die Notwendigkeit spürt, sich zusammenzutun, und mit Menschen zusammenzuarbeiten, die ihre Sorgen teilen." Erich Fromm, deutsch-amerikanischer Psychoanalytiker (1900-1980)

"Meine Hauptlehre aber ist vorläufig diese:

der Vater sorge für sein Haus, der Handwerker für seine Kunden, der Geistliche für gegenseitige Liebe, und die Polizei störe die Freude nicht."

Johann Wolfgang von Goethe, deutscher Dichter (1749-1832) 